\title{
TEST DE AUTOEVALUACIÓN
}

\section{Verifique sus conocimientos complementarias (I)}

Jordi Galimany

Enfermero. Profesor Asociado de la Escuela Universitaria de Enfermería de la Universidad de Barcelona. Supervisor del servicio de diagnósticos en la clínica Teknon. Barcelona. España.

EN LOS ÚLTIMOS AÑOS las exploraciones complementarias y las pruebas diagnósticas han experimentado una importante transformación. El papel de los profesionales de enfermería en este entorno es básico para satisfacer las necesidades del paciente en este tipo de actuación sanitaria, tanto como elementos informadores sobre los procedimientos y preparaciones, como para llevar a cabo las posibles atenciones/curas posteriores al procedimiento diagnóstico.

Este primer artículo pretende ser un acercamiento, que se completará con una serie de otros específicos sobre cada grupo de exploraciones.

1. ¿A qué nos referimos cuando, en el ámbito sanitario, hablamos de exploraciones o pruebas complementarias?

a. Exploraciones que curan al paciente.

b. Estudios que únicamente sirven para diagnosticar patologías.

c. Pruebas que se realizan en los centros sanitarios de alto nivel para satisfacer la tranquilidad de pacientes y familiares.

d. En todo caso son exploraciones que complementan un diagnóstico clínico.

e. Ninguna es correcta.

2. En el terreno del diagnóstico por la imagen contamos con diferentes disciplinas para llegar a una resolución diagnóstica:

a. Estudios radiológicos simples o convencionales.

b. Ecografía (ultrasonidos). c. Resonancia magnética (RM)

d. Medicina nuclear y algunas disciplinas más recientes, como la tomografía por emisión de positrones (PET).

e. Todas son ciertas.

3. ¿Para qué sirven las pruebas diagnósticas?

a. Para diagnosticar.

b. Para curar.

c. Para efectuar el seguimiento evolutivo de enfermedades.

d. Para el cribado (screening) de la población de riesgo.

e. Todas son ciertas.

4. ¿Qué deberíamos saber sobre el proceso de realización de una exploración diagnóstica?

a. No es una responsabilidad del profesional de enfermería. b. Deberíamos tener en cuenta la preparación de la prueba.

c. Deberíamos tener en cuenta la preparación de la prueba, y las curas posteriores a dicha prueba.

d. Deberíamos explicar con detalle en qué consiste la exploración, tener en cuenta la preparación de la prueba y las posibles curas posteriores.

e. Hay que tener en cuenta que las exploraciones se hacen en el servicio de radiología y, por tanto, todo depende de ellos.

5. En la preparación de la prueba, es cierto que:

a. Cuanto menos le expliquemos, más ayudamos a tranquilizar al paciente.

b. La preparación no suele tener mucha importancia.

c. Al explicar al paciente la dinámica de la prueba reducimos su ansiedad ante la misma.

d. Si el paciente sabe en qué consiste la exploración o prueba, facilitamos su colaboración.

e. Son ciertas c y d.

6. ¿Qué consideramos como preparación de la exploración diagnóstica?

a. La preparación consiste siempre en estar en ayuno.

b. La preparación consiste siempre en estar en ayuno y tomar laxantes unas horas antes de la prueba. 
c. La explicación de qué le harán durante la prueba.

d. La preparación física, si procede (ayuno, limpieza intestinal, medicación, etc.), y consentimiento informado con explicación detallada de la prueba.

e. Ninguna es correcta.

7. En el proceso posprocedimiento diagnóstico es cierto que:

a. No es problema nuestro, lo hace todo el médico.

b. Lo más importante son las curas posprocedimiento.

c. Hay que tener presente las curas posprocedimiento, explicar los signos y síntomas derivados de la técnica e intentar, dentro de nuestros medios, orientar sobre el tiempo necesario de espera para conocer el resultado de la prueba.

d. Debemos explicar los signos y síntomas derivados de la técnica.

e. Todas son ciertas.

8. Las pruebas complementarias:

a. Son el complemento a la exploración física del paciente.

b. Complementan el juicio clínico que realiza el facultativo.

c. Tienen diferentes ramas: análisis clínicos, pruebas funcionales y estudios morfológicos.

d. Los estudios morfológicos pueden ser de tipo endoscópico y laparoscópico, y de diagnóstico por la imagen.

e. Todas son ciertas.
9. Las pruebas de diagnóstico por la imagen:

a. Utilizan todas rayos $\mathrm{X}$ en alguna de sus fases.

b. Ninguna utiliza rayos $X$.

c. La radiología convencional y la tomografía computarizada (TC) utilizan rayos $X$.

d. Utilizan rayos X: la radiología convencional, la radiología contrastada, la TC, la mamografía y la densitometría ósea, aunque de baja intensidad.

e. Las respuestas c y d son correctas.

10. En los procedimientos de diagnóstico por la imagen:

a. La ecografía se basa en el uso de ultrasonidos para llegar a un diagnóstico.

b. La RM es un procedimiento que no utiliza radiación ionizante.

c. Las técnicas de medicina nuclear utilizan materiales radiactivos.

d. La PET es una técnica de medicina nuclear.

e. Todas son ciertas.

11. ¿A qué nos referimos cuando en diagnóstico por la imagen hablamos de técnicas terapéuticas?

a. A que al someter a muchas exploraciones a un paciente podemos resolver algunos procesos patológicos.

b. A que algunos procedimientos de radiología intervencionista pueden ser también terapéuticos. c. A que algunos procedimientos, en principio diagnósticos, pueden servir para realizar punciones, drenajes $u$ otras intervenciones curativas.

d. A que ayudándonos de procesos de imagen diagnóstica podemos llegar a un diagnóstico clínico sin más.

e. Las respuestas b y c son correctas.

12. Los contrastes utilizados en diagnóstico con radiación ionizante:

a. Siempre son productos o fármacos radiactivos.

b. Los contrastes radiológicos no son radiactivos, se utilizan medios de contraste oral e intravenoso.

c. Nunca se utilizan medios de contraste en radiología.

d. Las técnicas de contraste hacen referencia a isótopos y sólo se utilizan en medicina nuclear.

e. Todas son falsas.

13. Los contrastes que se utilizan en las pruebas de radiología:

a. Son inocuos.

b. Son sustancias que ayudan a descartar estructuras anatómicas.

c. Siempre se administran por vía intravenosa.

d. Siempre se administran por vía oral.

e. Ninguna de las respuestas es correcta. 
14. En los estudios diagnósticos, el consentimiento informado hace referencia a:

a. La necesidad de que el paciente esté informado de los beneficios de la prueba.

b. La necesidad de que el paciente reciba un informe detallado del diagnóstico una vez realizado.

c. Al derecho que tiene el paciente o sus familiares a recibir información sobre los riesgos y alternativas al procedimiento.

d. Al derecho del paciente a no recibir información referente al procedimiento que le van a realizar.

e. Las respuestas a, c y d son correctas.

15. ¿Cuál de las siguientes

afirmaciones no es cierta?

a. Actualmente se realizan menos exploraciones complementarias.

b. Actualmente se realizan más exploraciones complementarias.

c. Las exploraciones complementarias no sustituyen a la exploración clínica, que es imprescindible.

d. Las exploraciones deben estar indicadas según el protocolo de indicación correspondiente.

e. Las técnicas diagnósticas están muy ligadas a los avances tecnológicos.

\section{Respuestas}

1. d. Cuando en el ámbito sanitario se habla de exploraciones o pruebas complementarias, se hace referencia a exploraciones que complementan, como su nombre indica, un diagnóstico clínico. Está claro que las exploraciones complementarias no pueden curar por sí solas; aunque en algunos casos, como veremos más adelante, pueden aplicarse procedimientos terapéuticos. De este modo tampoco es cierto que únicamente sirvan para diagnosticar, y cada vez tienen más utilidad en el campo del intervencionismo y, por tanto, pueden considerarse curativas o terapéuticas. Otro tema es la problemática de la demanda de exploraciones complementarias para satisfacer al paciente o para cubrir un espacio de protección legal de los profesionales facultativos; no creemos que sea el motivo esencial por el cual se solicitan exploraciones complementarias, aunque a veces así lo parezca.

2. e. En el terreno del diagnóstico por la imagen contamos con diferentes disciplinas para llegar a una resolución diagnóstica: los estudios radiológicos simples -también llamados de radiología convencional-, los estudios basados en los ultrasonidos -conocidos como ecografías-, más recientemente la RM, las técnicas de medicina nuclear que se basan en el uso de isótopos radiactivos y algunas disciplinas todavía más recientes, como la PET. Los estudios de radiología con contrastes, la tomografía computarizada (TC) y las técnicas hemodinámicas completan esta lista. Todas estas disciplinas pueden encuadrarse en los servicios de diagnóstico por la imagen de los centros sanitarios.

3. e. Las pruebas diagnósticas sirven para establecer el diagnóstico inicial de la patología, para confirmar un diagnóstico clínico o completarlo, para hacer seguimiento evolutivo de las enfermedades y como estudio preoperatorio o como pretratamiento. Aunque en menor medida y cada vez más, las pruebas complementarias también se utilizan como mecanismo curativo o terapéutico. Esto se debe a la posibilidad de realizar técnicas menos invasivas que requieren menos infraestructura sanitaria y que permiten una convalecencia sustancialmente menor.

Lamentablemente, en algunas situaciones derivadas de la práctica clínica diaria las pruebas complementarias se solicitan de manera inapropiada con el fin de satisfacer la demanda de atención que exige el paciente o su entorno. No se debería caer en el error de ceder a esas presiones.

4. d. En cuanto al proceso de realización de una exploración diagnóstica, el profesional de enfermería debería saber explicar con detalle en qué consiste la exploración, teniendo en cuenta que tanto la preparación como las posibles curas posteriores también forman parte de ella. Éste es un punto crucial, ya que el profesional de enfermería es el profesional sanitario más próximo al enfermo y con más contacto con él.

5. e. La explicación de la dinámica de la prueba contribuye a reducir la ansiedad del paciente. Esto permite que el paciente colabore en la preparación previa, en el desarrollo de la misma y en las curas posteriores que deberemos atender. Además contribuirá a que pierda los posibles miedos generados por el desconocimiento del procedimiento diagnóstico.

\section{6. d. La preparación correcta de la} prueba comprende una parte física y una parte de información. La parte física dependerá, en gran manera, de qué tipo de exploración se trata. En muchos casos la preparación no es necesaria o es prácticamente irrelevante (radiología convencional o simple), mientras que en otros casos el hecho de no hacerla de manera correcta puede impedir la realización de la técnica (pruebas con contraste). También debe tenerse en cuenta que en algunos casos -afortunadamente pocos- la no correcta preparación puede entrañar un riesgo para el paciente (caso de técnicas intervencionistas que requieran determinaciones analíticas previas de coagulación o de estado de la función renal).

En lo que se refiere a la información que debe suministrarse al paciente, está por una parte la que nosotros, como profesionales de enfermería, debemos facilitarle por lo mencionado en las respuestas anteriores, y por otra la que contempla el consentimiento informado en cualquier procedimiento. Esta última está sujeta a consideraciones legales que implican la necesidad de que la persona que realice la técnica tenga claro y entienda qué se le hará y qué riesgos conlleva.

7. c. En pacientes hospitalizados, o en el caso de llevar a cabo nuestra labor profesional en un departamento de diagnóstico, hay que tener presente las curas que se realizan después de la 
técnica, que pueden ser inmediatas después de la prueba o realizarse más posteriormente.

Las técnicas intervencionistas tienen cada vez más relevancia, y esto implica un seguimiento posterior fuera del área diagnóstica por parte de los profesionales de enfermería. En este contexto debemos conocer los signos y síntomas derivados de la técnica. Si es posible, hay que intentar orientar sobre el tiempo que suele tardar el resultado de la prueba, ya que contribuye en gran medida a la tranquilidad del paciente. A la hora de obtener/proporcionar esta información no se deben traspasar los límites de nuestra competencia y recordar que el tiempo de espera depende de múltiples factores que no siempre conocemos o controlamos.

En las técnicas a pacientes no hospitalizados o ambulatorios nuestro campo de acción se limita a las posibles consultas posteriores una vez realizada la prueba.

8. e. Las pruebas complementarias, tal como su nombre indica, son un complemento al juicio clínico en la exploración física del paciente. En el contexto del diagnóstico de la enfermedad, las pruebas complementarias pueden dividirse en 3 grandes apartados: a) análisis clínicos; b) estudios morfológicos, y c) pruebas funcionales. Los análisis clínicos comprenden la inmunología, la anatomía patológica, la bioquímica y la microbiología. En nuestro caso nos centraremos en el apartado de los estudios morfológicos, que pueden dividirse en técnicas endoscópicas o laparoscópicas y en técnicas de diagnóstico por la imagen. El diagnóstico por la imagen lo componen la radiología convencional y contrastada, la mamografía, la TC, la densitometría, la ecografía, la RM, la medicina nuclear y, más recientemente, la PET.

Por otro lado, el grupo de pruebas funcionales lo forman, entre otros, las pruebas de esfuerzo, las pruebas funcionales respiratorias, la electromiografía, el electrocardiograma, el electroencefalograma, la urodinámica, etc.
9. e. Las técnicas de diagnóstico que basan la obtención de una imagen diagnóstica en la radiación ionizante son: la radiología convencional y contrastada, la mamografía, la TC y la densitometría ósea; utilizan radiación $\mathrm{X}$ o radiación ionizante, lo que habitualmente denominamos rayos $\mathrm{X}$. Como salvedad, cabe decir que la densitometría ósea utiliza radiación ionizante de baja intensidad para determinar la densidad del hueso de las zonas de estudio, que suelen ser la cabeza femoral y los cuerpos vertebrales lumbares.

10. e. La gama de procedimientos de que disponemos para establecer el diagnóstico a partir de técnicas de imagen es cada vez más amplia. Por un lado contamos con las técnicas que utilizan radiación ionizante que hemos mencionado anteriormente; pero aunque éstas son las más usuales y con las que más habitualmente tomaremos contacto en la práctica clínica, no son las únicas que debemos conocer.

Las técnicas ecográficas, que se basan en la emisión de ultrasonidos de un cristal piezoeléctrico, constituyen un importante grupo de pruebas diagnósticas tanto desde el punto de vista cualitativo como cuantitativo.

Las exploraciones de RM son otro de los grupos importantes de diagnóstico. En los últimos años, además, han tomado una gran relevancia y se están produciendo innovaciones de manera casi constante, y estas técnicas se irán instaurando cada vez más y sustituirán a otras más invasivas o cruentas y que proporcionan menos información diagnóstica.

Las técnicas de medicina nuclear son ampliamente utilizadas en múltiples aspectos clínicos. Se han visto reforzadas por nuevas técnicas aplicadas al diagnóstico de patología cardíaca y oncología. Además, en los últimos tiempos la PET ha revolucionado las técnicas tradicionales de imagen, sobre todo en el campo de la oncología, ya que con la administración y rastreo de isótopos radiactivos detecta actividad tumoral incluso antes de que tenga traducción radiológica.
11. e. Cuando en diagnóstico por la imagen hablamos de técnicas terapéuticas, nos referimos tanto a procedimientos de radiología intervencionista que pueden ser diagnósticos y a la vez terapéuticos (como la radiología vascular, que diagnostica y trata lesiones de los vasos de forma percutánea, o un enema opaco en el caso de una invaginación intestinal, ya que diagnostica y reduce la invaginación) y también a procedimientos que pueden servir específicamente para la terapéutica: realizar drenajes y tratar lesiones tumorales mediante radiofrecuencia. Esto es fácil de entender, ya que si decimos que determinada lesión o estructura se visualiza correctamente mediante determinada técnica de imagen, es lógico que, si es necesario intervenir en dicha lesión -ya sea para obtener una muestra para cultivo, para eliminar o drenar dicha estructura, o para paliar procesos que la comprometan- se utilice esa técnica de imagen que permite visualizar dicha estructura anatómica.

No es cierto, sin embargo, en el caso de imagen diagnóstica que realizar muchas exploraciones sea curativo; la radiación curativa pertenece al campo de la radioterapia y no es el caso de este artículo.

También es conveniente recordar en este punto que las técnicas diagnósticas, aunque en algún caso sean curativas, no resuelven por sí solas el juicio o el diagnóstico clínico y no lo sustituyen.

12. d. Los medios de contraste en radiología se utilizan para rellenar cavidades naturales, para determinar y estudiar su morfología y dinámica. Pueden ser muy variados, dependiendo de la técnica utilizada, y suponen una herramienta básica.

Por un lado están los que se utilizan para la visualización del tracto digestivo en radiología (sulfato de bario), que pueden administrarse por vía oral o rectal. Por otro lado contamos con los contrastes yodados, que pueden administrarse por vía oral diluidos o por vía intravenosa; se utilizan básicamente 
para visualizar el sistema genitourinario y el sistema vascular, y se usan en radiología, en la TC y en hemodinámica.

Hay otro tipo de contrastes que se utilizan en estudios de RM y que son específicos de dicha técnica (contrastes paramagnéticos).

Ninguno de los anteriores es radiactivo; no emiten radiación.

Las sustancias radiactivas son las que se utilizan en medicina nuclear.

13. e. Los contrastes utilizados en radiología no son, por desgracia, inocuos. Aunque las reacciones adversas se han reducido de manera sustancial en los últimos años, debemos seguir contemplándolas como una de las complicaciones en la realización de pruebas radiológicas.

Igual que cualquier otro fármaco o sustancia que administramos en el organismo, sea por vía oral, rectal o intravenosa, los contrastes pueden provocar efectos indeseados (reacciones alérgicas, insuficiencia renal).

Tanto desde un punto de vista cualitativo como cuantitativo, los contrastes yodados intravenosos son los que pueden provocar más reacciones adversas. Los contrastes con base de sulfatos de bario pueden provocar alteraciones menos graves, como estreñimiento o alteraciones en el aspecto de las heces. Los contrastes paramagnéticos son a priori poco alergógenos; sus principales manifestaciones en pacientes sensibles son mareos y náuseas, y no se han descrito reacciones graves.
14. e. El documento del consentimiento informado no es exclusivo de las exploraciones diagnósticas. Igual que en el resto de procedimientos medicosanitarios, pretende asegurar que el paciente o sus familiares tengan toda la información referente al procedimiento o prueba. En ningún caso exime de responsabilidad en la realización del mismo a los profesionales sanitarios. La ley establece en cada caso cómo y cuándo debe realizarse este consentimiento informado. Como norma general, ello corresponde al facultativo prescriptor de la exploración, aunque nosotros, como profesionales de enfermería en contacto constante con el paciente y la familia, podemos ser requeridos para informar sobre las distintas exploraciones, su preparación, sus efectos secundarios o las posibles alternativas.

15. a. No es cierto que actualmente se realicen menos exploraciones o procedimientos diagnósticos. Lo que ocurre es precisamente todo lo contrario: cada vez se realizan más procedimientos diagnósticos en los hospitales y centros asistenciales. Esto, que en principio no debería ser un inconveniente, puede repercutir en la correcta praxis por parte de los profesionales sanitarios y generar problemas de costes y de presión en la demanda de dichas exploraciones. Las técnicas actuales son cada vez más sofisticadas y van ligadas a los avances tecnológicos, y aunque en algunos casos contribuyen a disminuir la práctica de otros procedimientos más costosos, su sofisticación provoca encarecimiento. La premisa de seguir las indicaciones para prescribir las técnicas y no usarlas como recurso de protección contra posibles demandas o como sustitutivo del juicio clínico es otro de los elementos que podrían debatirse, aunque éste no sea el marco adecuado. En todo caso, en el contexto actual la demanda de exploraciones no parece reducirse, sino que parece cada vez más un recurso para la resolución del proceso de enfermedad en sí mismo y no un recurso complementario, como parece lógico que fuera.

\section{Bibliografía}

Casanova R, Pedrosa CS. Diagnóstico por la imagen. Madrid: Interamericana; 1987

Eisenberg RL, Dennis CA. Radiología patológica. Madrid: Mosby-Year Book; 1992.

Fucks AW. Principles of radiographic exposure and processing. Florida: Springfield; 1964.

Greenfield GB, Cooper SJ. Manual de posiciones radiológicas. Barcelona: Jims; 1975.

Reith EJ, Braindenbach B, Lorenc M. Texto básico de anatomía y fisiología para enfermería. Barcelona: Doyma; 1990.

Swallow RA, Naylor E, Rosebuck EJ, Whitley AS Clark. Barcelona: Salvat; 1988

Varios autores. Atlas de anatomía radiológica. Barcelona: Doyma; 1990.

\section{Web de interés}

http://acir.net/

http://radiographics.rsnajnls.org/

http://radiology.creighton.edu/

http://www.acr.org/s_acr/index.asp

http://www.aetr.net/

http://www.ajronline.org/

http://www.alar-dxi.org/

http://www.csn.es/

http://www.ctisus.com/

http://www.eurorad.org/

http://www.radquiz.com/

http://www.seram.org/ 